\title{
Remarks on Measurable Sets and Functions
}

\author{
Roy O. Davies ${ }^{1}$
}

(October 19, 1965)

\begin{abstract}
A. J. Goldman (On measurable sets and functions, J. Res. NBS 69B (Math. and Math. Phys.) Nos. 1 and 2, 99-100 (1965)) conjectured that the Borel sets are characterized by their property of having measurable inverse images under all Lebesgue measurable functions: here it is pointed out that the existence of analytic non-Borel sets refutes this and a related conjecture. Also an error in Goldman's Theorem 2 is corrected.
\end{abstract}

Key Words: Measure, integration, real function.

We deal exclusively with subsets of the real line $R$, and with real-valued functions having $R$ as domain. Let $(B S)$ and $(B F)$ denote the respective families of Borel sets and Borel-measurable functions, while $(L S)$ and $(L F)$ denote the respective families of Lebesgue-measurable sets and functions. Then $f \epsilon(L F)$ if and only if

$$
f^{-1}(B) \epsilon(L S) \quad \text { for all } B \epsilon(B S) .
$$

Recently Goldman ${ }^{2}$ asked whether (1) characterized $(B S)$, in the sense of the following

Conjecture: If $\mathrm{S}$ is not in (BS), then there is an $\mathrm{f} \epsilon(\mathrm{LF})$ such that $\mathrm{f}^{-1}(\mathrm{~S})$ is not in (LS).

We can disprove this conjecture as follows. Let

$$
n=\{n(1), n(2), \ldots\}
$$

be generic notation for an infinite sequence of positive integers. If $\mathfrak{s}$ is a family of sets, then any set

$$
\bigcup_{n} \bigcap_{r=1}^{\infty} F(n(1), \ldots ., n(r))
$$

where each $F(n(1), \ldots, n(r)) \epsilon \tilde{F}$, is said to be "obtained from $\mathfrak{F}$ by operation $(\mathscr{A}) "$. If $\mathscr{A}(\mathfrak{F})$ consists of all sets obtainable from $\mathfrak{s}$ by operation $(\mathscr{A})$, then for any function $f$,

$$
f^{-1}(\mathscr{A}(\mathfrak{F}))=\mathscr{A}\left(f^{-1}(\mathfrak{F})\right)
$$

\footnotetext{
${ }^{1}$ Department of Mathematics, The University, Leicester, United Kingdom.

${ }^{2}$ A. J. Goldman, On measurable sets and functions, J. Res. NBS 69B (Math. and Math. Phys.) Nos. 1 and 2, 99-100 (1965).
}

When $\mathfrak{i}=(B S), \mathscr{A}(\mathfrak{s})$ is called the class of analytic sets, and it is known ${ }^{3}$ that

$$
(B S) \subset \mathscr{A}(B S) \text { but }(B S) \neq \mathscr{A}(B S) \text {. }
$$

For any $f \epsilon(L F)$, it follows from (1) and (2) that

$$
f^{-1}(\mathscr{A}(B S)) \subset \mathscr{A}(L S) .
$$

It is also known ${ }^{4}$ that $(L S)$ is closed under operation $(\mathscr{A})$, so that (4) implies

$$
f^{-1}(\mathscr{A}(B S)) \subset(L S) \quad \text { for all } f \epsilon(L F) .
$$

Considering $S \epsilon \mathscr{A}(B S)-(B S)$, as permitted by (3), we are led via (5) to a contradiction of the conjecture.

Denote functional composition by an asterisk $((f * g)(x)=f(g(x)))$, and let $(L C F)$ be the class of functions $f$ such that

$$
g \epsilon(L F) \text { implies } f^{*} * g \epsilon(L F) .
$$

Goldman (Theorem 4, op cit) also showed that we should have

$$
(B F)=(L C F)
$$

if the Conjecture were true. That (6) fails together with the Conjecture can be proved by choosing as $f$ the characteristic function of some $S \epsilon \mathscr{A}(B S)-(B S)$; clearly $f$ is not in $(B F)$, but for any $B \epsilon(B S)$ we have $f^{-1}(B)$ a member of $\mathscr{A}(B S)$, namely $R$ or $\phi$ or $S$ or $R-S$,

\footnotetext{
${ }^{3}$ K. Kuratowski, Topologie I, 2d ed. (Warsaw, 1948), p. 391

${ }^{4}$ K. Kuratowski, op. cit., p. 64.
} 
so that for any $g \epsilon(L F)$ it follows from (5) that

$$
(f * g)^{-1}(B)=g^{-1}\left(f^{-1}(B)\right) \epsilon(L S),
$$

proving $f * g \epsilon(L F)$ and hence $f \epsilon(L C F)$.

Thus the problem of finding a satisfactory characterization of $(L C F)$ remains open. If $(Q S)$ is the class of sets $Q$ such that

$$
g^{-1}(Q) \epsilon(L S) \quad \text { for all } g \epsilon(L F),
$$

then $f \epsilon(L C F)$ if and only if

$$
f^{-1}(B) \epsilon(Q S) \quad \text { for all } B \epsilon(B S) .
$$

Hence characterizing $(L C F)$ is closely related to characterizing $(Q S)$.
Finally, Goldman's Theorem 2 (op cit) should be amended to read as follows:

THEOREM: For any $\mathrm{B} \epsilon(\mathrm{BS})$ and $\mathrm{L} \epsilon(\mathrm{LS})$, with sole exceptions $(\mathrm{B}=\phi, \mathrm{L} \neq \phi)$ and $(\mathrm{B}=\mathrm{R}, \mathrm{L} \neq \mathrm{R})$, there is an $\mathrm{f} \epsilon(\mathrm{LF})$ such that $\mathrm{L}=\mathrm{f}^{-1}(\mathrm{~B})$.

Proof: If $B=\phi$ and $L=\phi$, or $B=R$ and $L=R$, then any $f \epsilon(L F)$ will do. If $B=\phi$ and $L \neq \phi$, or $B=R$ and $L \neq R$, then no $f$ will do. Finally, if $B \neq \phi$ and $B \neq R$, then we can define $f$ on $L$ so that $f(L) \subset B$, and on $R-L$ so that $f(R-L) \subset R-B$. 